\title{
A new model of field-aligned currents derived from high-precision satellite magnetic field data
}

\author{
V. O. Papitashvili, ${ }^{1}$ F. Christiansen, and T. Neubert ${ }^{2}$ \\ Solar-Terrestrial Physics Division, Danish Meteorological Institute, Copenhagen, Denmark \\ Received 10 October 2001; revised 20 December 2001; accepted 28 December 2001; published 23 July 2002.
}

[1] A model of field-aligned currents is derived from highprecision magnetic field measurements from the Ørsted and Magsat satellites, being parameterized by the interplanetary magnetic field strength and direction for summer, winter and equinox. The high-precision data allow the model to be determined directly by a simple 2-D curl technique combined with fitting of spherical harmonic functions. New elements of the model are: (a) the FAC patterns are determined separately for both polar regions, resolving the seasonal dependence of interhemispheric asymmetries, (b) the IMF $\sim 0$, ground-state patterns are also resolved; these elements are obtained for the first time. From the model, the total upward/downward currents have been determined for various IMF conditions. The ratio of the summer/winter currents is $\sim 1.35$ and the equinox currents $\sim 1$. The model allows FAC mapping for IMF $|B| \leq 12 \mathrm{nT}$, except during magnetic storms and substorms. INDEX TERMS: 2471 Ionosphere: Plasma waves and instabilities; 2712 Magnetospheric Physics: Electric fields (2411); 2708 Magnetospheric Physics: Current systems (2409); 2409 Ionosphere: Current systems (2708)

\section{Introduction}

[2] One of the important elements in the chain of events from the Sun to Earth's environment is the currents flowing along magnetic field lines between the magnetopause and the upper atmosphere in the polar regions, transporting significant energy and momentum from the solar wind into the atmosphere. However, since the publication of the first statistical diagram of R1/R2 field-aligned currents derived from satellite data [Iijima and Potemra, 1976], no comprehensive studies of ionospheric FAC distributions were undertaken based on direct mesasurements in space until recently [e.g., Weimer, 2001]. In the interim period, the NBZ FAC system and $B_{y}$-controlled currents were identified, and FAC patterns and their dependence on IMF were inferred from inversion of ground magnetometer data.

[3] The model presented here is based on high-precision magnetic field survey data from the satellites Magsat [Langel and Estes, 1980] and Ørsted [Neubert et al., 2001]. Both satellites are in low-Earth polar orbit, with Magsat (1979-1980) in the dawn-dusk meridian and Ørsted (1999-present) slowly drifting in local time. Today, the combined dataset covers all local times and seasons in both

\footnotetext{
${ }^{1}$ Also at Space Physics Research Laboratory, University of Michigan, Ann Arbor, Michigan, USA.

${ }^{2}$ Now at Danish Space Research Institute, Copenhagen, Denmark.

hemispheres, with $\sim 26$ million samples above $\pm 45^{\circ}$ corrected geomagnetic latitudes.

[4] The method used to determine the FACs is based on Ampere's law. Magnetic perturbations above $\pm 45^{\circ}$ are first calculated subtracting the main field given by the DGRF1980 or Ørsted initial field model and then transformed into CGM coordinates. The data are further converted into Cartesian coordinates with $x$ directed towards dawn and $y$ towards noon, and binned in square cells of $1^{\circ}$ in latitude $(\sim 111 \mathrm{~km})$ according to IMF clock angle, intensity and seasons (summer, winter, and equinox). Assuming vertical field line geometry, the FAC density at a grid cell is determined as the curl of the horizontal magnetic disturbances and then smoothed over $5 \times 5$ grid-cells centered at the desired cell. Finally, the spatial grid of obtained FACs are fitted with spherical harmonic functions and continued downward to the ionospheric altitude $115 \mathrm{~km}$ using current conservation. By trial and error, we limited the expansion degree $n=21$ and the order $m=3$ to obtain the FAC patterns with the fewest terms.

\section{Results}

[5] The data used are from Magsat during November 1979-April 1980 ( 7.5 million samples) and from Ørsted from April 1999 through October 2001 ( 19 million samples), selecting all polar passes satisfying the IMF conditions: $B_{T}=\left(B_{y}^{2}+B_{z}^{2}\right)^{1 / 2} \leq 1 \mathrm{nT}($ median $\sim 0 \mathrm{nT})$, $1 \mathrm{nT}<B_{T} \leq 4 \mathrm{nT}$ (median $2.7 \mathrm{nT}$ ), and $B_{T}>4 \mathrm{nT}$ (median $\sim 5.7 \mathrm{nT}$ ), divided in eight IMF clock-angles as shown in Figure 1. The IMF data are obtained from IMP 8, ISEE, and ACE spacecraft and then ballistically propagated to the nominal magnetopause at $12 R_{E}$. The model is limited to $|B| \leq 12 \mathrm{nT}$ as data coverage is too poor above this value. The model is not valid for periods of rapid changes of the magnetosphere configuration as during magnetic storms and substorms.

[6] For each season and both hemispheres experimental FAC distributions are obtained for each IMF sector (Figure A1 in the electronic appendix). The 17 distributions representing one hemisphere and one season were fitted by spherical harmonic functions. The FAC distribution for an arbitrary IMF angle and intensity is determined from a linear fit to the coefficients representing the three IMF median magnitudes along the four main clock angles: $0^{\circ}, 90^{\circ}, 180^{\circ}$, and $270^{\circ}$. This gives the ionospheric FAC responses to changes in the IMF $B_{z}$ and $B_{y}$ components separately.

[7] Figure 2(A2) shows how the model captures the basic FAC patterns. The IMF $\sim 0$ and $B_{z}=-1 \mathrm{nT}$ currents display the R1/R2 FAC system, with some currents also in the near- 


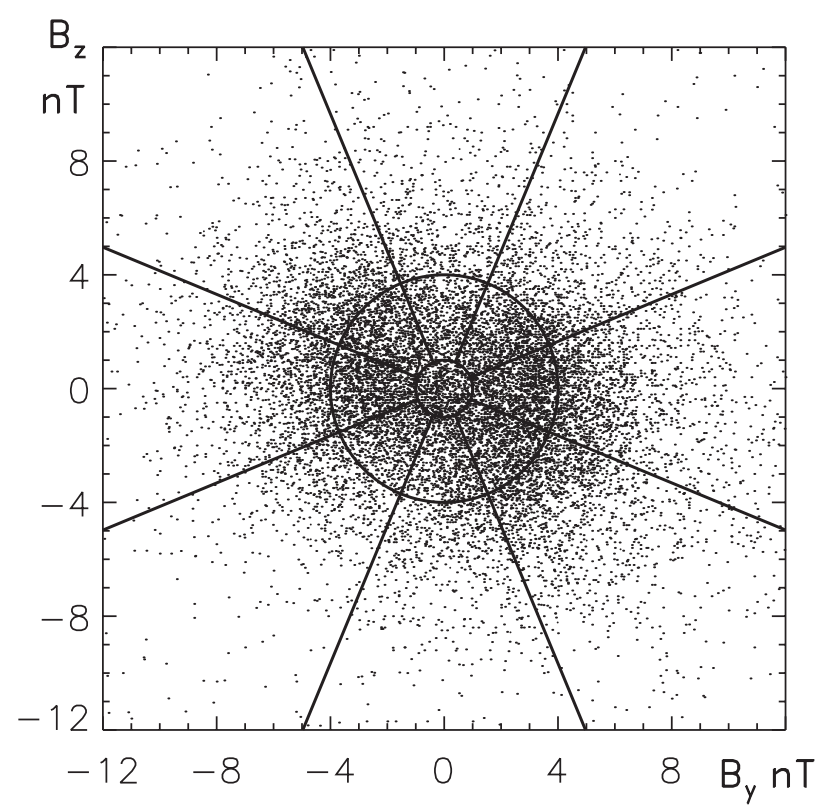

Figure 1. Distribution of IMF values used in this study and the bins in the IMF clock angle and intensity. The plot is limited to $\left|B_{z}\right|=\left|B_{y}\right|=12 \mathrm{nT}$ because of poor distribution of larger IMF values.

pole region [e.g., Yamauchi and Araki, 1989; Papitashvili et al., 2001]. The $B_{z}=+1 \mathrm{nT}$ maps show the NBZ current system, which is stronger in the sunlit polar cap. The $B_{y^{-}}$ controlled currents show significant seasonal asymmetry, while exhibiting the Svalgaard-Mansurov effect with upward (downward) currents in the center of the northern (southern) polar cap.

[8] The IMF clock-angle dependence is shown in Figure 3 (A3a) for $B_{T}=5 \mathrm{nT}$ over the northern winter and southern summer polar regions (other seasons are shown in Figure A3). The maps show the expected features of the high-latitude ionospheric FACs. The R1/R2 system is the dominant feature even in the dark, winter hemisphere. The equinox patterns
(Figure A3b) are very symmetric between hemispheres, showing almost no differences in the current intensities. The FACs are much stronger in the sunlit polar cap (Figure A3a,c).

\section{Discussion}

[9] While detailed comparisons with other data and other models have not yet been undertaken, the first results of the model presented here seem to be in general agreement with our understanding of the FAC patterns. The integrated hemispherical upward and downward current densities are shown in Figures 3 and A3. In Table 1, the ratios between the total upward/downward currents in the northern and southern polar regions are summarized for southward IMF clock-angles. This allows a rough estimation of the seasonal dependence of R1/R2 currents which dominate during southward IMF. As seen, the total currents are about symmetrically distributed between the two hemispheres during equinox, while the summer currents are $\sim 1.35$ times stronger than the winter currents. This is in good agreement with our recent findings [Christiansen et al., 2002].

[10] In approach, our model is most closely related to the model developed recently by Weimer [2001] from DE 2 satellite magnetometer data. The Weimer model provides FAC maps over a polar ionosphere parameterized by the IMF and dipole tilt, but it does not explicitly contain the IMF $\sim 0$, ground-state patterns and does not resolve the two polar regions separately. Figure 4 shows the scatter plot of FACs min/max densities from Weimer's Figures 1, 3, 4, and our Figures 3 and A3. One can see that both models respond similarly to the IMF changes, although the plotted linear fit shows $\sim 25 \%$ differences between the two modeled currents.

[11] We understand that comparisons of all upward/ downward FACs (total or limited to certain sectors) would make better physical sense; unfortunately, Weimer did not calculate these currents. Note that while his model is based on more sophisticated data processing, our model utilizes a simple technique but shows similar results because it is based on better quality data. The most important part is that

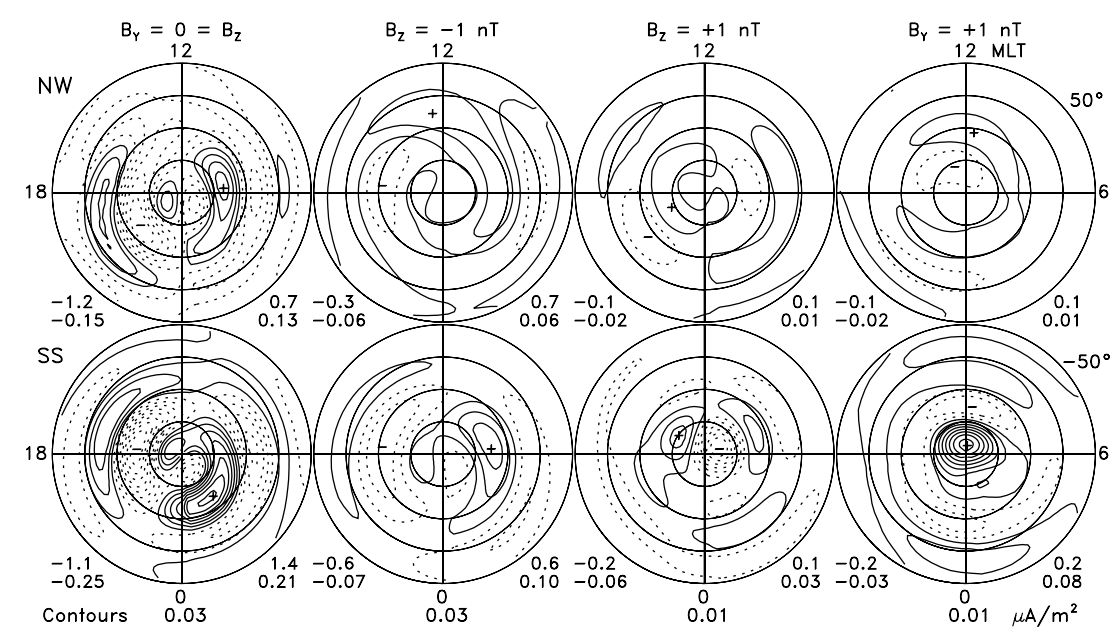

Figure 2. Modeled basic FACs patterns for northern winter (top) and southern summer (bottom) for IMF marked on top. The leftmost plot is the "ground state" FAC pattern for $B_{T}=\left(B_{y}^{2}+B_{z}^{2}\right)^{1 / 2}=0 \mathrm{nT}$. The other patterns are the responses per $1 \mathrm{nT}$ of $B_{T} \neq 0$ and should be added to the ground state pattern. The contour intervals are indicated at the bottom; note that zero contours are not plotted. Labels are as on Figure 3. 


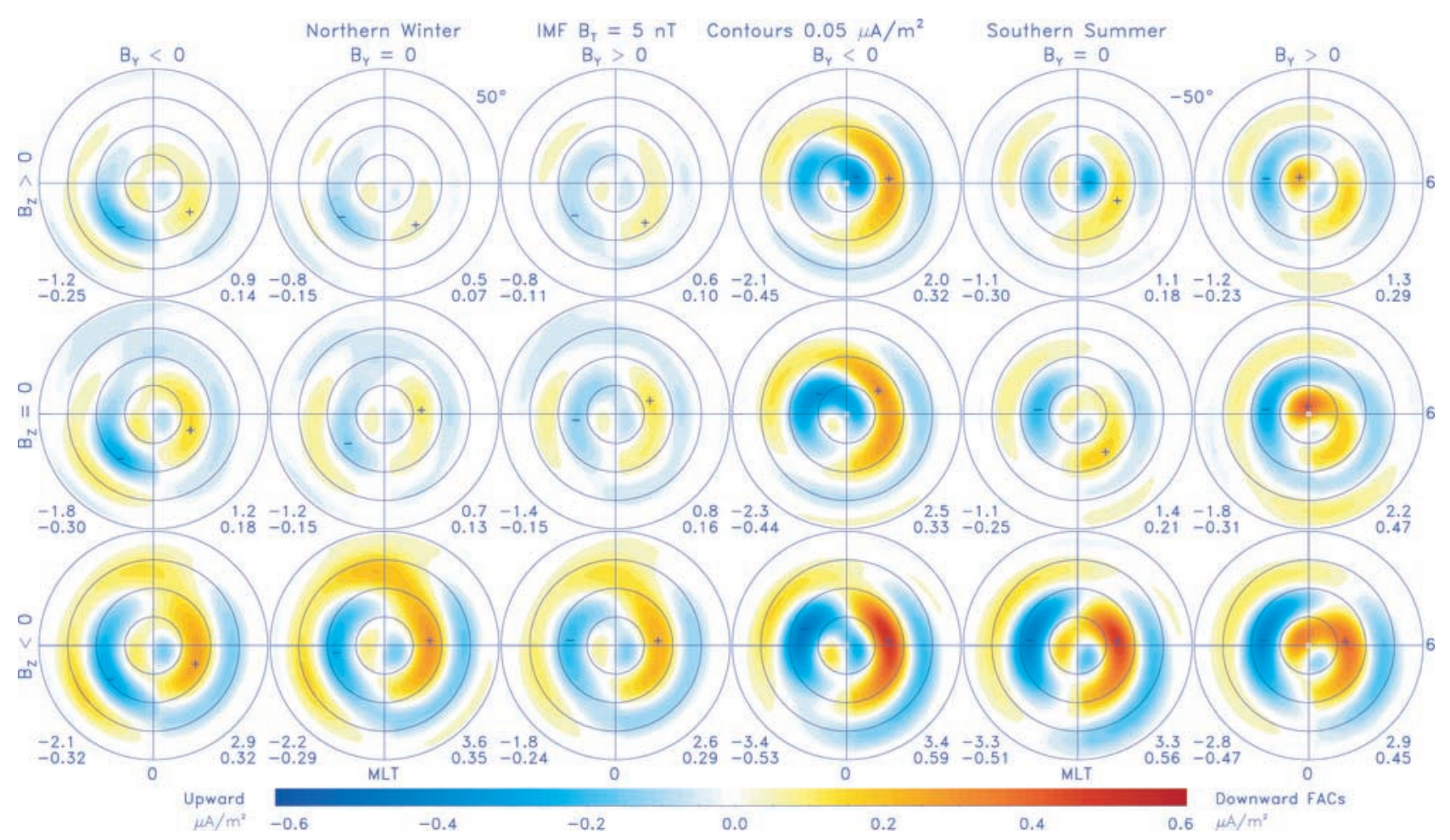

Figure 3. Maps of field-aligned currents for $B_{T}=5 \mathrm{nT}$ organized by the IMF clock angle for northern winter (left) and southern summer (right). The central plots are the patterns for $B_{T}=0$. The total hemispherical currents (top numbers, MA) and the minimum and maximum current densities (lower numbers, $\mu \mathrm{A} / \mathrm{m}^{2}$ ) are marked at the bottom corners of each polar subplot. The upward currents are negative and downward - positive. The FACs min/max density locations are identified by "_" and "+".

these similar results were obtained with entirely different data sets, thereby providing an independent verification of the other results. It is particularly interesting that there are minor features in some of the FAC patterns (particularly near the poles) that one might think are artifacts of the modeling process, yet these features appear in both models.

[12] Another interesting approach of special relevance to real-time monitoring of global FAC distributions is the use of engineering magnetometer data on the 66 Iridium satellites [Waters et al., 2001]. Their hourly averaged map in Figure 3 matches well with our FAC pattern for the IMF values representing the hour $\left(B_{y}=-5.6 \mathrm{nT}, B_{z}=-5.9 \mathrm{nT}\right)$, modeled through our Web-based interfaces http://www.dmi.dk/ projects/oersted/face.html or http://www.sprl.umich.edu/ mist/limie.html.

[13] In conclusion, we note that a new FAC model has been developed based on high-precision magnetic field data taken from low-Earth orbit spacecraft. The model is parameterized by the IMF strength and direction and provides

Table 1. Interhemispheric Summer/Winter and Equinox Ratios for Total Upward/Downward FACs and Southward IMF Clock Angles from Figures 3 and A3

\begin{tabular}{lccccccc}
\hline IMF & \multicolumn{2}{c}{$225^{\circ}$} & \multicolumn{2}{c}{$180^{\circ}$} & \multicolumn{2}{c}{$135^{\circ}$} & Aver. \\
Pol.Cap & $\uparrow$ & $\downarrow$ & $\uparrow$ & $\downarrow$ & $\uparrow$ & $\downarrow$ & Ratio \\
\hline NS/SW & 1.44 & 1.23 & 1.31 & 1.27 & 1.46 & 1.57 & 1.38 \\
NE/SE & 1.00 & 1.03 & 1.06 & 0.97 & 1.00 & 1.00 & 1.00 \\
SS/NW & 1.62 & 1.17 & 1.50 & 0.92 & 1.56 & 1.12 & 1.32 \\
\hline
\end{tabular}

maps of currents explicitly for the two polar regions. It allows studies of different components of the global FAC system, such as the R1, R2 and the dayside and nightside currents. The new model shows that the ionospheric FAC patterns are much more complex than the canonical R1/R2

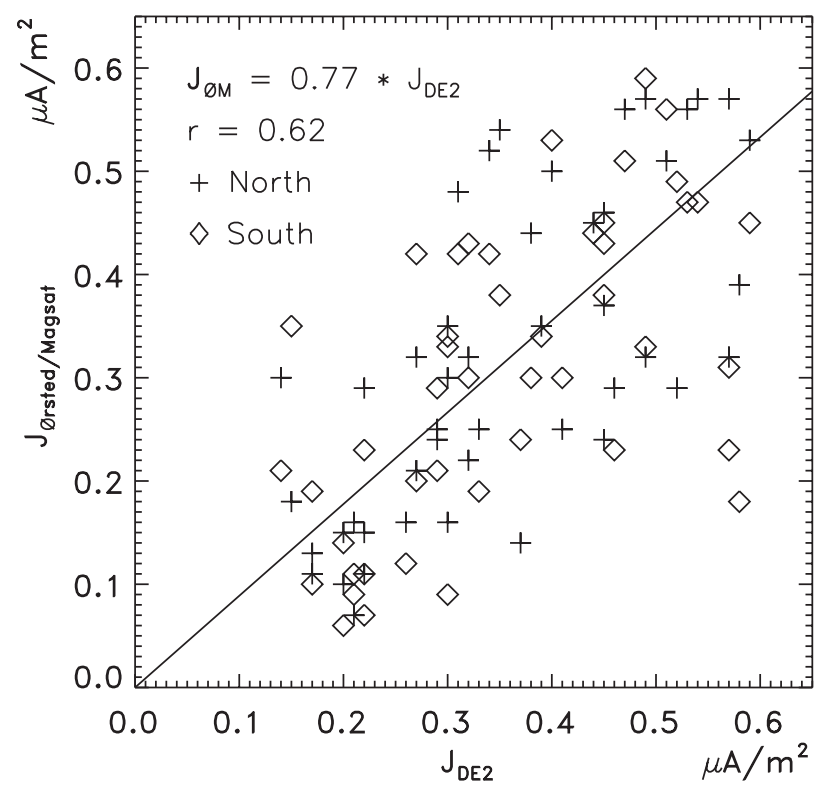

Figure 4. Comparison of absolute FACs min/max densities taken from Weimer [2001] and our Figures 3(A3). 
system of Iijima and Potemra, and it is important that the space physics community learns about the new paradigm.

[14] Acknowledgments. The IMF data used in this study were obtained from the NASA's NSSDC and the NOAA's SEC Web sites. We are grateful for the support of the Ørsted Project provided by the Danish Ministries of Transport, Research and Information Technology, and Trade and Industry. Additional support to the Ørsted Project was provided by NASA, ESA, CNES, and DARA. In Michigan, the work was supported by the NSF/ATM-9727554, 9980075, and DoD/AFOSR F49620.01-1-0359.

\section{References}

Christiansen F., V. O. Papitashvili, and T. Neubert, Seasonal variations of high-latitude field-aligned current systems inferred from Ørsted and Magsat observations, J. Geophys. Res., 107(A2), DOI 10.1029/ 2001JA900104, 2002.

Iijima, T., and T. A. Potemra, Field-aligned currents in the dayside cusp observed by Triad, J. Geophys. Res., 81, 5971, 1976.

Langel, R. A., and R. H. Estes, The near-Earth magnetic field at 1980 determined from Magsat data, J. Geophys. Res., 90, 2495, 1980.
Neubert, T., M. Mandea, and G. Hulot, et al., Ørsted satellite captures high-precision geomagnetic field data, Eos Trans. $A G U, 82(7), 81$, 2001.

Papitashvili, V. O., F. Christiansen, and T. Neubert, Field-aligned currents during IMF $\sim$ 0, Geophys. Res. Lett., 28, 3055, 2001.

Waters, C. L., B. J. Anderson, and F. Liou, Estimation of global field aligned currents using the Iridium system magnetometer data, Geophys. Res. Lett., 28, 2165, 2001.

Weimer, D. R., Maps of ionospheric field-aligned currents as a function of the interplanetary magnetic field derived from Dynamic Explorer 2 data, J. Geophys. Res., 106, 12,889, 2001.

Yamauchi, M., and T. Araki, The interplanetary magnetic field $B_{y}$-dependent field-aligned currents in the dayside polar cap under quiet conditions, J. Geophys. Res., 94, 2684, 1989.

F. Christiansen, T. Neubert, and V. O. Papitashvili, Solar-Terrestrial Physics Division, Danish Meteorological Institute, Lyngbyvej 100, Copenhagen, DK-2100, Denmark. (fch@dmi.dk; neubert@dsri.dk; papita@, umich.edu) 